\title{
Inverse planning for combination of intracavitary and interstitial brachytherapy for locally advanced cervical cancer
}

\author{
Kotaro YOSHIO ${ }^{1, *}$, Naoya MURAKAMI ${ }^{1}$, Madoka MOROTA ${ }^{1}$, Ken HARADA ${ }^{1}$, \\ Mayuka KITAGUCHI ${ }^{1}$, Kentaro YAMAGISHI ${ }^{1}$, Shuhei SEKII ${ }^{1}$, Kana TAKAHASHI ${ }^{1}$, Koji INABA ${ }^{1}$, \\ Hiroshi MAYAHARA ${ }^{1}$, Yoshinori ITO ${ }^{1}$, Minako SUMI ${ }^{1}$, Susumu KANAZAWA ${ }^{2}$ and Jun ITAMI ${ }^{1}$ \\ ${ }^{1}$ Department of Radiation Oncology, National Cancer Center Hospital, Tokyo, Japan \\ ${ }^{2}$ Department of Radiology, Okayama University Hospital, Okayama, Japan \\ *Corresponding author. Department of Radiation Oncology, National Cancer Center Hospital, 5-1-1 Tsukiji Chuo-ku, Tokyo, \\ 104-0045, Japan. Tel: + 81-3-3542-2511; Fax: + 81-3-3545-3567; Email: ko.taro1201@ gmail.com
}

(Received 19 October 2012; revised 24 April 2013; accepted 25 April 2013)

\begin{abstract}
The main purpose of this study was to compare three different treatment plans for locally advanced cervical cancer: (i) the inverse-planning simulated annealing (IPSA) plan for combination brachytherapy (BT) of interstitial and intracavitary brachytherapy, (ii) manual optimization based on the Manchester system for combination-BT, and (iii) the conventional Manchester system using only tandem and ovoids. This was a retrospective study of 25 consecutive implants. The high-risk clinical target volume (HR-CTV) and organs at risk were defined according to the GEC-ESTRO Working Group definitions. A dose of 6 Gy was prescribed. The uniform cost function for dose constraints was applied to all IPSA-generated plans. The coverage of the HR-CTV by IPSA for combination-BT was equivalent to that of manual optimization, and was better than that of the Manchester system using only tandem and ovoids. The mean $\mathrm{V}_{100}$ achieved by IPSA for combinationBT, manual optimization and Manchester was $96 \pm 3.7 \%, 95 \pm 5.5 \%$ and $80 \pm 13.4 \%$, respectively. The mean $\mathrm{D}_{100}$ was $483 \pm 80,487 \pm 97$ and $335 \pm 119 \mathrm{cGy}$, respectively. The mean $\mathrm{D}_{90}$ was $677 \pm 61,681 \pm 88$ and $513 \pm 150 \mathrm{cGy}$, respectively. IPSA resulted in significant reductions of the doses to the rectum (IPSA $\mathrm{D}_{2 \mathrm{~cm}^{3}}$ : $408 \pm 71 \mathrm{cGy}$ vs manual optimization $\mathrm{D}_{2 \mathrm{~cm}^{3}}: 485 \pm 105 \mathrm{cGy} ; P=0.03$ ) and the bladder (IPSA $\mathrm{D}_{2 \mathrm{~cm}^{3}}$ : $452 \pm 60 \mathrm{cGy}$ vs manual optimization $\mathrm{D}_{2 \mathrm{~cm}}$ : $\left.583 \pm 113 \mathrm{cGy} ; P<0.0001\right)$. In conclusion, combination-BT achieved better tumor coverage, and plans using IPSA provided significant sparing of normal tissues without compromising CTV coverage.
\end{abstract}

Keywords: cervical cancer; IPSA; combination brachytherapy; optimize; HDR

\section{INTRODUCTION}

Intracavitary brachytherapy (ICBT) plays a major role in the treatment of patients with cervical carcinoma [1-5]. When a tumor cannot be optimally encompassed by standard ICBT, interstitial brachytherapy (ISBT) is recommended to achieve better dose distribution [6].

In our hospital, combination brachytherapy (BT) of ICBT and ISBT has been performed to achieve even better dose distribution for advanced bulky lesions. However, an appropriate optimizing method for combination-BT has not been established. Until recently we performed manual optimization based on the Manchester system.
Inverse-planning simulated annealing (IPSA) is an optimization tool of high-dose rate brachytherapy (HDR-BT) that has been developed at the University of California, San Francisco [7, 8]. The optimal solution is obtained by minimizing the objective function through an iterative process. The algorithm uses fast simulated annealing to process the cost functions to arrive at an optimal solution in less than a minute. IPSA has been found to be superior with respect to target coverage and normal-tissue sparing compared with traditional optimization methods for prostate [9-13] and gynecologic malignancies [7, 14-16].

The main purpose of this study is to compare three different treatment plans for locally advanced cervical cancer: 
(i) the IPSA plan for combination-BT, (ii) manual optimization based on the Manchester system for combination-BT, and (iii) the conventional Manchester system using only tandem and ovoids.

\section{MATERIALS AND METHODS}

This is a retrospective study of 25 consecutive implants (for nine patients) for primary cervical cancers. All patients selected for this study underwent conventional external radiation therapy and HDR-BT using combination-BT between August 2010 and March 2012. All patients were classified into Stage IIIB according to the International Federation of Gynecology and Obstetrics (FIGO) system. With external radiation therapy using 15-MV X-rays in a conventional fractionation, the whole pelvis was irradiated by up to $30 \mathrm{~Gy}$, and the ensuing 20 Gy to the pelvic sidewall was administered with central shielding. Combination-BT was initiated upon the introduction of the central shielding. External radiation therapy was not performed on the day of BT. For combination-BT, CT-compatible tandem and ovoids were used with plastic interstitial needles (5F Proguide needles; Nucletron BV, Veenendaal, Netherlands). They were inserted under transrectal ultrasound guidance and fluoroscopy without a template (Fig. 1). Combination-BT was performed under caudal and local anesthesia. The interstitial catheters were placed to encircle the parametrial invasion. The distance between catheters was kept less than $2 \mathrm{~cm}$, if possible. After the insertion, CT scans of 2-mm slice thickness were taken on an Aquilion LB CT scanner (TOSHIBA Medical Systems, Japan). After CT scanning, needle positions were changed if we could not make a favorable dose distribution, or if the needle position was close to the organs at risk (OARs). The depth of the needle was determined by

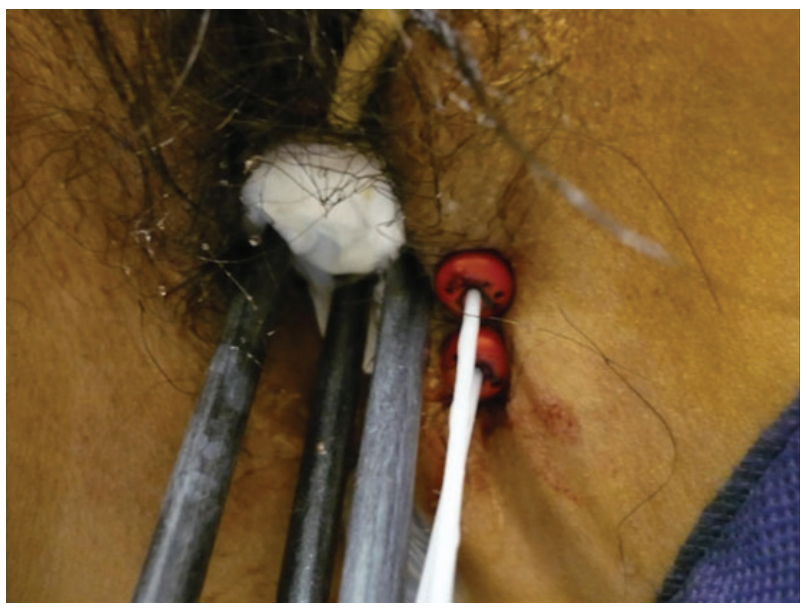

Fig. 1. Two plastic interstitial needles are inserted from the left side of vulva. A CT-compatible tandem and ovoids are also inserted.
CT images. Because the off-set length of plastic needle is $4 \mathrm{~mm}$, we need to take the off-set length into consideration to make a favorable dose distribution. Treatment planning was based on post-implant CT imaging and was performed using Oncentra v4.1 (Nucletron, The Netherlands). A radiation oncologist delineated the high-risk clinical target volume (HR-CTV) using pretreatment clinical extent, imaging (pretreatment and during external radiation therapy, but just before the start of BT), intraoperative findings, and radioopaque silver markers placed during the procedure. HR-CTV volumes were defined according to the GEC-ESTRO Working Group recommendation [17]. OAR volumes included the bladder, rectum, bowel and vagina. The rectal wall and its cavity were contoured from the anus to the rectosigmoid flexure. All bowel walls and their cavity surrounding the uterus and $2 \mathrm{~cm}$ above the fundus of the uterus were contoured. The vagina wall was drawn with $2-\mathrm{mm}$ thickness. All treatments were performed with the plan produced by manual optimization based on the Manchester system with a prescribed dose of $6 \mathrm{~Gy} /$ fraction. All applicators were removed after combination-BT and the procedure was repeated for each session of combination-BT. All patients underwent four fractions of HDR-BT with a total prescribed dose of 24 Gy. For comparison with manual optimization, HDR dose distributions were created according to the different dose optimization methods as follows: the conventional Manchester system using only tandem and ovoids; IPSA using combination-BT; and IPSA using only tandem and ovoids. Figure 2 shows the dose distributions achieved by each planning method.

\section{Conventional Manchester system}

For the production of manual optimization, conventional ICBT with only tandem and ovoids was made according to the Manchester system. In the Manchester system, the dose to point $\mathrm{A}$ was set to $6 \mathrm{~Gy}$, and the relative contribution of tandem and ovoids to the point $\mathrm{A}$ dose was set to 1.8:1.

\section{Manual optimization based on the Manchester system}

After the production of the conventional Manchester dose planning, the locations of dwell position were determined for the interstitial applicator based on CT images. Coronal and sagittal images were very useful. Each dwell position was set with 5-mm intervals. About $20 \%$ of the ovoid's dwell time was allocated to the interstitial applicators. Finally, finetuning was performed by manual graphical optimization to realize adequate dose coverage to the HR-CTV. Basically, dwell times of dwell points in each interstitial applicator were set up equally in this method. Point A was irradiated at more than 6 Gy in this method. 

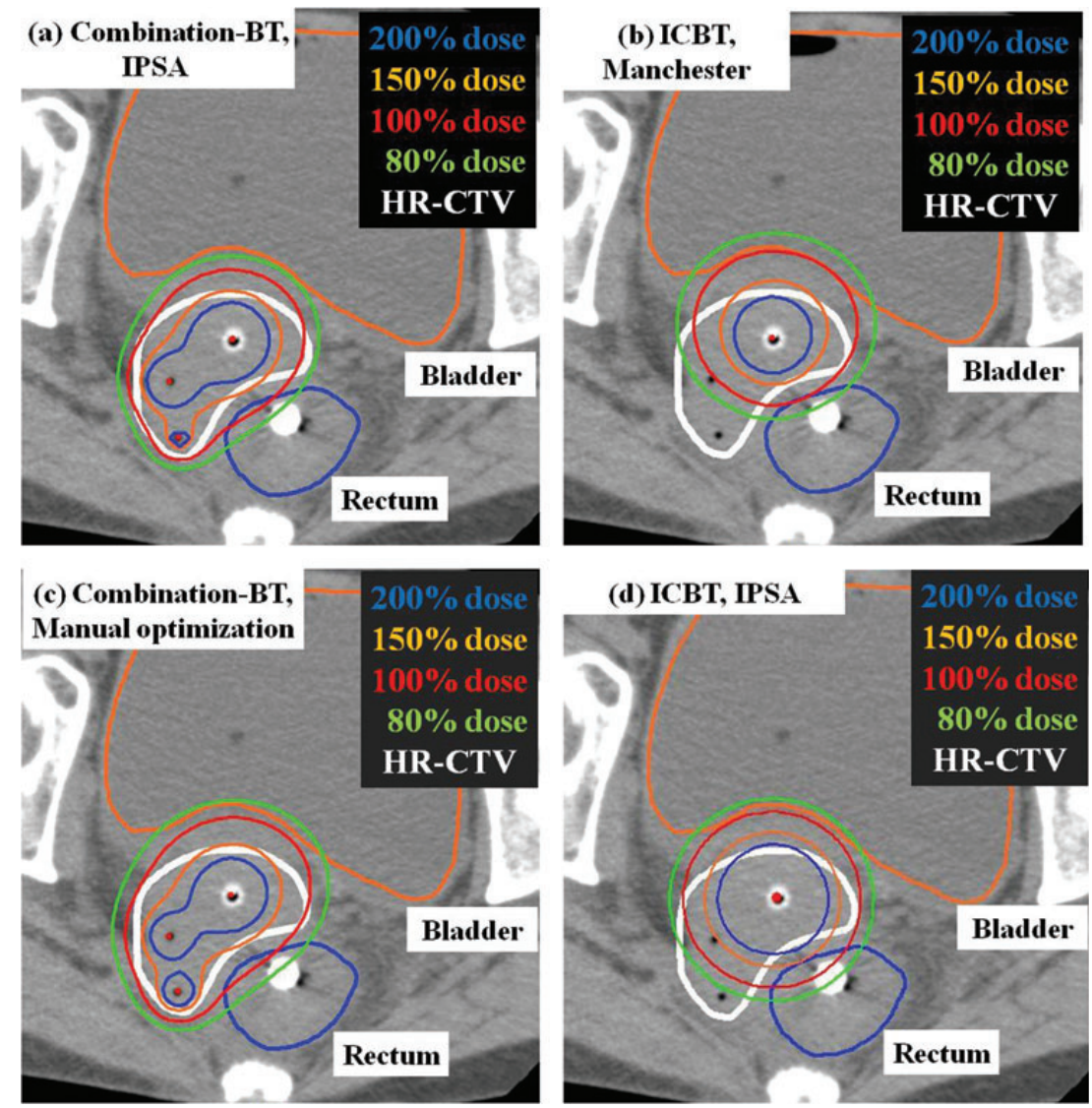

Fig. 2. Comparison of dose distributions between three planning methods: (a) Inverse planning simulated annealing (IPSA) for combination-BT, (b) conventional Manchester system using tandem and ovoids, and (c) manual optimization based on the Manchester system for combination-BT, as well as (d) IPSA using tandem and ovoids.

\section{IPSA}

In IPSA, an optimal plan is sought that meets the dose objective parameters of both CTV and the OARs with the parameters' relative importance represented by weights. Both optimal dwell positions and dwell times are calculated. In this study, about 20-second sets of IPSA were repeated up to three times. Table 1 provides a representative set of dose objective parameters used to obtain an IPSA plan in this study. IPSA with combination-BT and ICBT used the same dose objective parameter. The weight is a factor reflecting the relative importance.

\section{Plan evaluation}

Dosimetric outcomes from the four different plans were compared. We analyzed the dose-volume histograms with the following endpoints: $\mathrm{D}_{100}$ and $\mathrm{D}_{90}$ for the minimum doses to $100 \%$ and $90 \%$ volumes of HR-CTV, and $\mathrm{V}_{200}, \mathrm{~V}_{150}$ and $\mathrm{V}_{100}$ for the volumes of HR-CTV enclosed by 200, 150 and $100 \%$ of the prescribed dose of combination-BT. The volumes covered by $100 \%\left(\mathrm{~V}_{\mathrm{PD}}\right)$ and $200 \%\left(\mathrm{~V}_{2 \mathrm{PD}}\right)$ of the prescription dose were also evaluated. Bladder, rectum, bowel and vagina were considered as OARs. For each organ, $\mathrm{D}_{0.1 \mathrm{~cm}^{3}}, \mathrm{D}_{1 \mathrm{~cm}^{3}}$, and $\mathrm{D}_{2 \mathrm{~cm}^{3}}$ were calculated for the maximal doses to $0.1 \mathrm{~cm}^{3}, 1 \mathrm{~cm}^{3}$ and $2 \mathrm{~cm}^{3}$ of the OARs, respectively. The homogeneity index (HI) was defined as (V100-V150)/ V100. The differences between the parameters were compared for the four plans using analysis of variance (ANOVA), with resultant two-sided $P$ values $<0.05$ considered to indicate statistically significant differences.

\section{RESULTS}

All 25 implants of the nine patients were evaluated. Their median age was 51 years (range, 43-63). The mean number of implanted interstitial needles was 2.8 (range, 2-6). The mean HR-CTV was $29.6 \mathrm{ml}$ (range, 5.4-66.3).

\section{Dose and volume parameters for HR-CTV and $\mathbf{V}_{\text {PD }}$ (Table 2)}

A summary of the dose and volume parameters for OARs (bladder, rectum, bowel and vagina) is shown in Table 2 . Coverage of the HR-CTV with IPSA and manual 
Table 1. Representative set of dose objective parameters used in IPSA

\begin{tabular}{lcccc}
\hline & $\begin{array}{c}\text { Minimum } \\
(\mathbf{c G y})\end{array}$ & Weight & $\begin{array}{c}\text { Maximum } \\
(\mathbf{c G y})\end{array}$ & Weight \\
\hline $\begin{array}{l}\text { HR-CTV } \\
\text { surface }\end{array}$ & 600 & 200 & & \\
$\begin{array}{l}\text { HR-CTV } \\
\quad \text { volume }\end{array}$ & 600 & 100 & & \\
$\begin{array}{l}\text { Bladder surface } \\
\text { Rectum surface }\end{array}$ & & & 450 & 100 \\
Bowel surface & & & 450 & 100 \\
\hline
\end{tabular}

HR-CTV $=$ high-risk clinical target volume, IPSA = inverseplanning simulated annealing.

optimization using combination-BT was significantly better than that with the conventional Manchester system of ICBT alone. HR-CTV coverage was the same for both IPSA and manual optimization of combination-BT with a mean $\mathrm{V}_{100}$ of $96 \pm 3.7$ and $95 \pm 5.5 \%$, respectively, a mean $D_{100}$ of $483 \pm 80$ and $487 \pm 97$ cGy, respectively, and a mean $\mathrm{D}_{90}$ of $677 \pm 61$ and $681 \pm 88 \mathrm{cGy}$, respectively. $\mathrm{V}_{100}, \mathrm{D}_{100}$ and $\mathrm{D}_{90}$ showed no significant difference between IPSA and manual optimization. In addition, despite the equivalent or better CTV coverage, the $\mathrm{V}_{\mathrm{PD}}$ and $\mathrm{V}_{2 \mathrm{PD}}$ volumes obtained by the IPSA of combination-BT (mean $\mathrm{V}_{\mathrm{PD}}, 89 \pm 33$ and $\mathrm{V}_{2 \mathrm{PD}}, 21 \pm 8.6 \mathrm{ml}$ ) were significantly lower than those obtained by manual optimization (mean $\mathrm{V}_{\mathrm{PD}}, 131 \pm 22$ and $\mathrm{V}_{2 \mathrm{PD}}, 30 \pm 6.5 \mathrm{ml}$ ) or the Manchester system of ICBT alone (mean $\mathrm{V}_{\mathrm{PD}}, 116 \pm 10$ and $\mathrm{V}_{2 \mathrm{PD}}, 29 \pm 4.6 \mathrm{ml}$ ). $\mathrm{V}_{150}, \mathrm{~V}_{200}$ and HI showed no significant difference between IPSA with combination-BT, manual optimization of combination-BT, and the Manchester system of ICBT alone. For reference, the dose-volume parameter achieved by IPSA using tandem and ovoids alone is also listed in Table 2.

\section{Dose and volume parameters for OARs (Table 2)}

The data suggest that IPSA with combination-BT leads to favorable sparing of the adjacent organs. The dose to the OARs obtained from IPSA with combination-BT (rectum $\mathrm{D}_{2 \mathrm{~cm}^{3}}$ : $408 \pm 71 \mathrm{cGy}$, bladder $\mathrm{D}_{2 \mathrm{~cm}^{3}}: 452 \pm 60 \mathrm{cGy}$, bowel $\mathrm{D}_{2 \mathrm{~cm}^{3}}: 345 \pm 105 \mathrm{cGy}$ ) was generally lower than for that of the other plans.

\section{DISCUSSION}

The results of the present study have shown that combination-BT is an effective method for achieving better dose distribution for advanced cervical lesions. Even manual optimization was able to bring about better coverage of the HR-CTV, with no significant increase of the dose to OARs in combination-BT. In addition, IPSA with combination-BT has resulted in significant sparing of normal tissues without compromising CTV coverage, compared with manual optimization.

Transperitoneal template techniques were commonly used as interstitial treatment methods for locally advanced tumor [18-21]. However, using these methods, the applicators have to be left for a few days after implantation. On the other hand, combination-BT is done for each brachytherapy session. The applicators do not need to be left and patients can be treated on an outpatient basis. In addition, there is no concern about interfractional differences in the needle positions, since the plastic applicators are inserted each time. Similar techniques have already been reported from Vienna University and Gunma University. Kirisits et al. [22] used their modified ring applicator. Their combined intracavitary and interstitial BT provides a prescription dose of up to 15 mm lateral of point A. Wakatsuki et al. [23] inserted a needle applicator from the vaginal vault inside the ovoid. Unlike the Vienna University technique, our technique does not need a particular applicator. In addition, our technique can place needles in a greater variety of positions and make various dose distributions adapted to the tumor shape, as in the method of Wakatsuki et al.

Comparison of IPSA with other optimizing methods, such as graphical optimization and dose-point optimization for interstitial template brachytherapy, has previously been reported [7, 14-16]. Most of the earlier investigations were carried out in prostate and cervical cancer, using a template technique [9-16]. Their reports described a significant sparing of normal tissues without compromising tumor coverage. Although there are no reports on an appropriate optimizing method for combination-BT, the same tendency is demonstrated in this study.

Although combination-BT and IPSA techniques result in superior plans in terms of HR-CTV coverage, and sparing normal tissues with ease, caution is recommended in the general application of IPSA. Because the number of applicators is limited, source dwell time in each applicator is longer than that with the template technique and more applicators. Therefore, combination-BT techniques will have a greater high-dose-volume than template techniques with more applicators. Some reports showed that complications increase with a greater $\mathrm{V}_{\mathrm{PD}}[24,25]$. Although the $\mathrm{V}_{\mathrm{PD}}$ and $\mathrm{V}_{2 \mathrm{PD}}$ were significantly less than using the conventional Manchester system, the location of the high-dose area differed from that in the conventional Manchester system. Some clinical reports have indicated tolerance and a good local control rate upon using IPSA. Thibault et al. [26] reported on their 43 patients' clinical experiences of ISBT using IPSA for a locally advanced population unsuitable for ICBT. The two-year local control (LC) rate for primary cancer was $87 \%$, and Grade 3/4 late morbidity occurred in 12 patients. Their high incidence of severe late toxicities was primarily related to vaginal necrosis. They believe that those complications 
Table 2. Dose-volume parameters of the conventional Manchester system, manual optimization, and IPSA

\begin{tabular}{|c|c|c|c|c|c|c|c|c|}
\hline Parameters & $\begin{array}{l}\text { (a) ICBT, IPSA } \\
(\text { mean } \pm \text { SD })\end{array}$ & $\begin{array}{c}\text { (b) ICBT, } \\
\text { Manchester } \\
(\text { mean } \pm \text { SD) }\end{array}$ & $\begin{array}{l}\text { (c) combination-BT, } \\
\text { manual optimization } \\
(\text { mean } \pm \text { SD })\end{array}$ & $\begin{array}{l}\text { (d) combination-BT, } \\
\text { IPSA (mean } \pm \text { SD) }\end{array}$ & $\begin{array}{l}P \text {-value } \\
\text { (a) vs (b) }\end{array}$ & $\begin{array}{l}P \text {-value } \\
\text { (b) vs (c) }\end{array}$ & $\begin{array}{l}P \text {-value } \\
\text { (d) vs (b) }\end{array}$ & $\begin{array}{l}P \text {-value } \\
\text { (c) vs (d) }\end{array}$ \\
\hline \multicolumn{9}{|c|}{ High-Risk CTV } \\
\hline $\mathrm{V}_{200 \%}$ & $41.5( \pm 10.8)$ & $32.6( \pm 12.2)$ & $34.4( \pm 12.8)$ & $33.1( \pm 10.3)$ & 0.04 & 0.95 & 0.99 & 0.98 \\
\hline $\mathrm{V}_{150 \%}$ & $61.6( \pm 11.1)$ & $53.5( \pm 15)$ & $60.8( \pm 15.1)$ & $58( \pm 12.6)$ & 0.16 & 0.23 & 0.65 & 0.88 \\
\hline $\mathrm{V}_{100 \%}$ & $87( \pm 7.9)$ & $80( \pm 13.4)$ & $95( \pm 5.5)$ & $96( \pm 3.7)$ & 0.04 & $<0.0001$ & $<0.0001$ & 0.91 \\
\hline $\mathrm{D}_{100}(\mathrm{~Gy})$ & $356( \pm 101)$ & $335( \pm 119)$ & $487( \pm 97)$ & $483( \pm 80)$ & 0.88 & $<0.0001$ & $<0.0001$ & 0.99 \\
\hline $\mathrm{D}_{90}(\mathrm{~Gy})$ & $573( \pm 117)$ & $513( \pm 150)$ & $681( \pm 88)$ & $677( \pm 61)$ & 0.22 & $<0.0001$ & $<0.0001$ & 0.99 \\
\hline \multicolumn{9}{|l|}{ Rectum } \\
\hline $\mathrm{D}_{0.1 \mathrm{~cm}} 3$ & $638( \pm 203)$ & $662( \pm 192)$ & $624( \pm 148)$ & $519( \pm 86)$ & 0.95 & 0.63 & 0.003 & 0.03 \\
\hline $\mathrm{D}_{1 \mathrm{~cm}} 3$ & $514( \pm 153)$ & $554( \pm 151)$ & $527( \pm 116)$ & $442( \pm 73)$ & 0.68 & 0.69 & 0.003 & 0.03 \\
\hline $\mathrm{D}_{2 \mathrm{~cm}} 3$ & $463( \pm 135)$ & $503( \pm 131)$ & $485( \pm 105)$ & $408( \pm 71)$ & 0.6 & 0.82 & 0.006 & 0.03 \\
\hline \multicolumn{9}{|l|}{ Bladder } \\
\hline $\mathrm{D}_{0.1 \mathrm{~cm}} 3$ & $737( \pm 161)$ & $751( \pm 161)$ & $788( \pm 222)$ & $599( \pm 104)$ & 0.99 & 0.71 & 0.006 & 0.0005 \\
\hline $\mathrm{D}_{1 \mathrm{~cm}} 3$ & $602( \pm 122)$ & $640( \pm 132)$ & $630( \pm 126)$ & $491( \pm 66)$ & 0.62 & 0.94 & $<0.0001$ & 0.0001 \\
\hline $\mathrm{D}_{2 \mathrm{~cm}} 3$ & $540( \pm 106)$ & $596( \pm 122)$ & $583( \pm 113)$ & $452( \pm 60)$ & 0.24 & 0.89 & $<0.0001$ & $<0.0001$ \\
\hline \multicolumn{9}{|l|}{ Bowel } \\
\hline $\mathrm{D}_{0.1 \mathrm{~cm}} 3$ & $524( \pm 159)$ & $876( \pm 1279)$ & $913( \pm 1321)$ & $480( \pm 185)$ & 0.54 & 0.01 & 0.1 & 0.08 \\
\hline $\mathrm{D}_{1 \mathrm{~cm}} 3$ & $413( \pm 114)$ & $538( \pm 249)$ & $566( \pm 264)$ & $380( \pm 113)$ & 0.12 & 0.004 & 0.0003 & 0.001 \\
\hline $\mathrm{D}_{2 \mathrm{~cm}} 3$ & $373( \pm 99)$ & $479( \pm 180)$ & $518( \pm 183)$ & $345( \pm 105)$ & 0.06 & 0.03 & $<0.0001$ & $<0.0001$ \\
\hline \multicolumn{9}{|l|}{ Vagina } \\
\hline $\mathrm{D}_{0.1 \mathrm{~cm}} 3$ & $1566( \pm 596)$ & $1267( \pm 163)$ & $1294( \pm 372)$ & $1096( \pm 331)$ & 0.04 & 0.7 & 0.03 & 0.02 \\
\hline $\mathrm{D}_{1 \mathrm{~cm}} 3$ & $948( \pm 320)$ & $1004( \pm 150)$ & $911( \pm 141)$ & $688( \pm 151)$ & 0.77 & 0.003 & $<0.0001$ & $<0.0001$ \\
\hline $\mathrm{D}_{2 \mathrm{~cm}} 3$ & $623( \pm 198)$ & $865( \pm 150)$ & $763( \pm 105)$ & $524( \pm 119)$ & $<0.0001$ & 0.0001 & $<0.0001$ & $<0.0001$ \\
\hline \multicolumn{9}{|c|}{ Volume parameter } \\
\hline $\mathrm{V}_{\mathrm{PD}}$ & $92( \pm 56)$ & $116( \pm 10)$ & $131( \pm 22)$ & $89( \pm 33)$ & 0.07 & 0.0002 & 0.0002 & $<0.0001$ \\
\hline $\mathrm{V}_{2 \mathrm{PD}}$ & $27( \pm 19)$ & $29( \pm 4.6)$ & $30( \pm 6.5)$ & $21( \pm 8.6)$ & 0.84 & 0.88 & $<0.0001$ & $<0.0001$ \\
\hline HI & $0.29( \pm 0.08)$ & $0.34( \pm 0.09)$ & $0.36( \pm 0.14)$ & $0.39( \pm 0.12)$ & 0.38 & 0.93 & 0.3 & 0.67 \\
\hline
\end{tabular}

IPSA = inverse planning annealing, CTV = clinical target volume, ICBT = intracavitary brachytherapy, $\mathrm{SD}=$ standard deviation, $\mathrm{V}_{\mathrm{PD}}=$ the absolute volume covered by $100 \%$ of the prescription dose, $\mathrm{V}_{2 \mathrm{PD}}=$ the absolute volume covered by $200 \%$ of the prescription dose, HI (Homogeneity Index) is defined as (V100 - V150)/V100. 
may have been related to the learning curve of inserting the needles. Kim et al. [27] reported on 51 patients' experiences. There were no toxicities of Grade 4 or greater, and the frequencies of Grade 3 acute and late toxicities were $4 \%$ and $2 \%$, respectively. Local recurrence developed in only two patients. Although their initial clinical outcomes were tolerable, careful attention should be paid to minimize the dose to normal tissues. In addition, longer follow-up on the side effects is needed for the application of combination-BT and IPSA in clinical practice.

\section{CONCLUSION}

In conclusion, to our knowledge, this is the first report on the employment of the IPSA technique in HDR combination-BT planning. Combination-BT achieved better tumor coverage and plans using IPSA and provided significant sparing of normal tissues without compromising CTV coverage compared with manual optimization.

\section{FUNDING}

Part of this study was financially supported by the Cancer Research Development Fund (23-A-13) of the National Cancer Center and the Cancer Clinical Research Fund of the Ministry of Welfare, Health and Labor.

\section{REFERENCES}

1. Nakano T, Kato S, Ohno T et al. Long-term results of highdose rate intracavitary brachytherapy for squamous cell carcinoma of the uterine cervix. Cancer 2005;103:92-101.

2. Nakano T, Ohno T, Ishikawa $\mathrm{H}$ et al. Current advancement in radiation therapy for uterine cervical cancer. $J$ Rad Res 2010;51:1-8.

3. Rose PG, Bundy BN. Chemoradiation for locally advanced cervical cancer: does it help? J Clin Oncol 2002;20:891-3.

4. Eifel PJ, Winter K, Morris M et al. Pelvic irradiation with concurrent chemotherapy versus pelvic and para-aortic irradiation for high-risk cervical cancer: an update of radiation therapy oncology group trial (RTOG) 90-01. J Clin Oncol 2004:22: 872-80.

5. Pearcey R, Brundage M, Drouin P et al. Phase III trial comparing radical radiotherapy with and without cisplatin chemotherapy in patients with advanced squamous cell cancer of the cervix. J Clin Oncol 2002;20:966-72.

6. Nag S, Erickson B, Thomadsen B et al. The American Brachytherapy Society recommendations for high-dose-rate brachytherapy for carcinoma of the cervix. Int J Radiat Oncol Biol Phys 2000;48:201-11.

7. Lessard E, Hsu IC, Pouliot J. Inverse planning for interstitial gynecologic template brachytherapy: truly anatomy-based planning. Int J Radiat Oncol Biol Phys 2002;54:1243-51.

8. Lessard E, Pouliot J. Inverse planning anatomy-based dose optimization for HDR brachytherapy of the prostate using fast simulated annealing algorithm and dedicated objective function. Med Phys 2001;28:773-9.

9. Hsu IC, Lessard E, Weinberg V et al. Comparison of inverse planning simulated annealing and geometrical optimization for prostate high dose rate brachytherapy. Brachytherapy 2004;3: 147-52.

10. Lachance B, Beliveau-Nadeau D, Lessard E et al. Early clinical experience with anatomy based inverse planning dose optimization for high dose rate boost of the prostate. Int $J$ Radiat Oncol Biol Phys 2002;54:86-100.

11. Yoshioka Y, Nishimura T, Kamata M et al. Evaluation of anatomy based dwell position and inverse optimization in high dose rate brachytherapy of prostate cancer: a dosimetric comparison to a conventional cylindrical dwell position, geometric optimization and dose point optimization. Radiother Oncol 2005;75:311-7.

12. Kolkman-Deurloo IK, Deleye XG, Jansen PP et al. Anatomy based inverse planning in HDR prostate brachytherapy. Radiother Oncol 2004;73:73-7.

13. Jacob D, Raben A, Sarkar A et al. Anatomy-based inverse planning simulated annealing optimization in high-dose-rate prostate brachytherapy: significant dosimetric advantage over other optimization techniques. Int J Radiat Oncol Biol Phys 2008;72:820-7.

14. Dewitt KD, Hsu IC, Speight J et al. 3D inverse treatment planning for the tandem and ovoid applicator in cervical cancer. Int J Radiat Oncol Biol Phys 2005;63:1270-4.

15. Morton GC, Sankreacha R, Halina P et al. A comparison of anatomy-based inverse planning with simulated annealing and graphical optimization for high-dose-rate prostate brachytherapy. Brachytherapy 2008;7:12-6.

16. Jamema SV, Sharma S, Mahantshetty U et al. Comparison of IPSA with dose-point optimization and manual optimization for interstitial template brachytherapy for gynecologic cancers. Brachytherapy 2011;10:306-12.

17. Pötter R, Haie-Meder C, Van Limbergen E et al. Recommendations from gynaecological (GYN) GEC ESTRO working group (II): concepts and terms in 3D image-based treatment planning in cervix cancer brachytherapy-3D dose volume parameters and aspects of 3D image-based anatomy, radiation physics, radiobiology. Radiother Oncol 2006;78: 67-77.

18. Syed AM, Puthawala AA, Abdelaziz NN et al. Long-term results of low-dose-rate interstitial-intracavitary brachytherapy in the treatment of carcinoma of the cervix. Int $J$ Radiat Oncol Biol Phys 2002;54:67-78.

19. Nag S, Martínez-Monge $\mathrm{R}$, Selman AE et al. Interstitial brachytherapy in the management of primary carcinoma of the cervix and vagina. Gynecol Oncol 1998;70: 27-32.

20. Kuipers T, Hoekstra CJ, van't Riet A et al. HDR brachytherapy applied to cervical carcinoma with moderate lateral expansion: modified principles of treatment. Radiother Oncol 2001;58: 25-30.

21. Erickson B, Gillin MT. Interstitial implantation of gynecologic malignancies. J Surg Oncol 1997;66:285-95.

22. Kirisits C, Lang S, Dimopoulos J. The Vienna applicator for combined intracavitary and interstitial brachytherapy of cervical cancer: design, application, treatment planning, and 
dosimetric results. Int J Radiat Oncol Biol Phys 2006;65: 624-30.

23. Wakatsuki M, Ohno T, Yoshida D. Intracavitary combined with CT-guided interstitial brachytherapy for locally advanced uterine cervical cancer: introduction of the technique and a case presentation. J Radiat Res 2011;52:54-8.

24. Crook JM, Esche BA, Chaplain G et al. Dose-volume analysis and the prevention of radiation sequelae in cervical cancer. Radiother Oncol 1987;8:321-32.

25. Barillot I, Horiot $\mathrm{J}$, Maingon $\mathrm{P}$ et al. Impact on treatment outcome and late effects of customized treatment planning in cervix carcinomas: baseline results to compare new strategies. Int J Radiat Oncol Biol Phys 2000;48:189-200.

26. Thibault I, Lavallée MC, Aubin S et al. Inverse-planned gynecologic high-dose-rate interstitial brachytherapy: clinical outcomes and dose-volume histogram analysis. Brachytherapy 2012;11:181-91.

27. Kim DH, Wang-Chesebro A, Weinberg V et al. High-dose rate brachytherapy using inverse planning simulated annealing for locoregionally advanced cervical cancer: a clinical report with 2-year follow-up. Int J Radiat Oncol Biol Phys 2009;75: 1329-34. 